\title{
Incidencia de la recomendación bibliográfica como técnica de animación a la lectura a los estudiantes de Educación Secundaria
}

\author{
Wilder A. Caururo Sánchez \\ wildgustin29@hotmail.com \\ Universidad Nacional «Santiago Antúnez de Mayolo». Huaraz, Perú. \\ Yvett Gonzales Ortiz \\ Universidad Nacional «Santiago Antúnez de Mayolo». Huaraz, Perú. \\ Rolando Roca Zarzoza \\ Universidad Nacional «Santiago Antúnez de Mayolo». Huaraz, Perú. \\ Fiorella Rosario del Castillo Palacios \\ Universidad Nacional «Santiago Antúnez de Mayolo». Huaraz, Perú.
}

\section{RESUMEN}

Analiza la incidencia de la recomendación bibliográfica como técnica de animación a la lectura en los estudiantes del 5to de secundaria de las instituciones educativas Albert Einstein y San Martín de Porras de la ciudad de Huaraz. El estudio por su finalidad es investigación aplicada; por el diseño es de tipo cuasi-experimental con grupo control y experimental. Los resultados obtenidos determinan la incidencia positiva de la técnica en el incremento del interés por la lectura que contribuye al fortalecimiento del hábito lector, elevar los niveles de diversificación del gusto lector, desarrollar actitudes positivas hacia la lectura y el disfrute; y que el estudiante asuma la lectura como una actividad placentera que le permita alcanzar realización personal cognitiva. La investigación se realizó en el año lectivo 2019 desde los meses de mayo a diciembre. Para el análisis de los datos derivados de las encuestas se recurrió al uso de la estadística descriptiva.

Palabras clave: Técnica de animación de lectura; Recomendación bibliográfica 


\title{
Incidence of the bibliographic recommendation as an animation technique for reading to students Secondary Education.
}

\begin{abstract}
It analyzes the incidence of bibliographic recommendations as a technique of reading animation in the students of 4th and 5th years of secondary education in the I.E.P. Albert Einstein de Huaraz and I.E.P. San Martin de Porras in 2016. The study corresponds to the descriptive, correlational and transversal type; The relation between the independent variable (the technique of the bibliographic recommendation) and the dependent variable (motivation for reading) was
\end{abstract}

Keywords: Reading animation technique; Bibliographic recommendation.

Artículo recibido: 19 enero 2020

Aceptado para publicación: 22 febrero

Correspondencia:wildgustin29@hotmail.com

Conflictos de Interés: Ninguna que declarar 


\section{INTRODUCCIÓN}

La lectura presenta serias dificultades al momento de emplearse como medio de animación. En una investigación realizada por Bobbio-Alvarez (2014) encontró que el proceso se entorpece por la falta de motivación en casa, sin modelos que imitar, por lo que resulta imprescindible volver a esas prácticas que enriquecen el hábito lector. Por otro lado, el trabajo, la motivación del docente por la lectura ejerce a su vez influencia. ¿Leerán bien los docentes? ¿Serán los mejores para animar esta actividad? El autor en mención considera que la programación secuenciada de leer en las aulas no significa siempre una actividad que guste y anime; por el contrario, es sobre todo un control, que no está relacionado a la edad y nivel de los estudiantes ni sus intereses particulares. Por ello debe existir la intención, el interés de leer cuando exista una relación emocional con la actividad. Sedano (2015) en su investigación acerca de leer en el aula, plantea algunas causas que originan la falta de interés del estudiante, preocupa la escasa concentración, debido al desinterés por la lectura; léxico inadecuado, manifestado en el déficit del aprendizaje y la comprensión del mismo. Finalmente, no se debe dejar de lado el clima familiar, pues cuando no se ha formado los hábitos de estudio y no hubo apoyo, esta habilidad se logrará con dificultades.

Para arribar a la definición de animación a la lectura, se irá paulatinamente, especificando los conceptos de lectura, animación y promoción. Las definiciones y conceptos son innumerables. Se destaca la de Millán (2000) para quien la lectura es la herramienta que permite apropiarse de la información en cualquier ámbito, por medio del cual el individuo se desarrolla y accede al conocimiento. Asimismo, la lectura es considerada un proceso interactivo que establece una relación entre el texto y el lector; el cual al incorporarlo y elaborarlo le da un significado que es propio de ese individuo y no de otro (Anderson y Pearsonm, 1984; Solé, 1995). Es un proceso constructivo en el lector y con él confluyen sus vivencias, conocimientos, intereses, motivaciones para obtener, de ese texto, un significado que es único. (Devetach, 2008). «La lectura va a posibilitar el desarrollo de actitudes como la capacidad crítica (imprescindible en la actual sociedad de la información globalizada), la solidaridad y la contemplación reflexiva que sin duda proporciona la optimización del desarrollo emocional» (López, 2004:263). Las modalidades de lecturas, según Cassany (2003) son diversas entre las usuales son informativas, formativas y recreativas. 
La promoción de la lectura significa todo tipo de actividades que impulsen la aprehensión de la misma y su importancia en la formación de todos los individuos y de la sociedad en su conjunto (Bermúdez y Núñez, 2012). Además, es un hacer constante que se respalda en una actividad planificada que busca resultados a largo plazo. Mientras, la animación a la lectura consiste en una actividad que propone el acercamiento y la profundización en los libros de una forma creativa, lúdica y placentera (Cerrillo, 2004). Es un acto consiente realizado para producir una un acercamiento efectivo e intelectual a un libro concreto, de forma que este contacto produzca una estimación genérica hacia el libro (Sarto, 1993; Domech, 1994), que permita al estudiante un comportamiento lector (Poslaniec, 1990). Según Higuera (2016) la animación a la lectura comprende estrategias y acciones dirigidas a crear un vínculo entre un material de lectura y un individuo o grupo. En general, cuando se habla de animación a la lectura, se piensa desde un contexto que tiene relación con la escuela o la biblioteca y dirige la atención del estudiante al texto, «que incluyen tanto las formas escritas tradicionales (libros, revistas, documentos y periódicos) como las presentaciones electrónicas (Internet, correo electrónico y los mensajes de texto; así como los textos incluidos en cine, vídeo o televisión (anuncios publicitarios y subtítulos)» (Mullis y otros, 2006:4); y, con un objetivo sobre todo recreativo, buscando generar el gusto por la lectura, a través de los afectos y emociones. Las estrategias que se aplican son de diferente naturaleza y pueden ser tantas como la imaginación y la creatividad del animador. Higuera (2016) afirma que las técnicas de animación de la lectura conducen hacia el proceso lector y su práctica mediante diversas actividades como lectura en voz alta, clubes de lectura, tertulias literarias, cafés literarios y talleres de recomendación bibliográfica. El componente más importante que envuelve este tipo de actividades generalmente es el contexto lúdico, pues se focaliza en que el individuo pueda generar su gusto por leer sin sentir la presión de la obligación impuesta por el entorno curricular (Domech, 1994).

Existen multiplicidad de técnicas de animación a la lectura, las que se ajustan a la realidad y circunstancias vividas por los estudiantes. En la investigación realizada se enfatiza fundamentalmente una que a juicio de los investigadores se ajusta adecuadamente al nivel de estudio y a la realidad socio-sicopedagógica del grupo muestral de estudio seleccionado: la recomendación bibliográfica. Dicha técnica parte del objetivo de animar a los estudiantes a la organización de una biblioteca personal (se han de considerar las 
dimensiones planteadas) a partir de las recomendaciones bibliográficas desarrolladas por docente y sus compañeros. Para la aplicación de dicha técnica el docente debe contar con un gran sentido de empatía y apertura ante las recomendaciones brindadas por los estudiantes; por lo cual debe abstenerse de emitir juicios que desvaloren la calidad de textos refrendados por los educandos. Esta técnica permite conocer la amplia gama de intereses de los alumnos, genera espacios de diálogo y debate sobre la naturaleza del contenido de los textos evidenciados por los mismos alumnos y afina su gusto lector a partir de la socialización de sus intereses comunes. En el caso del docente permite conocer de primera mano los hábitos e intereses de los estudiantes y reorientar su trabajo en consonancia con ello. La importancia de la recomendación bibliográfica radica en la participación activa de los estudiantes en la generación de interés y el incremento de la intensidad lectora diversa en ellos, así como desarrollar «la actitud y auto-conceptos positivos con respecto al hábito lector como medio de disfrute personal (Mullis y otros, 2003). Por otro lado, la técnica propuesta pone en los hombros del docente la necesidad de convertirse en un lector de amplios intereses que no disuene con la sintonía de lo seleccionado por los estudiantes. En muchos casos, implica una asunción y examen desprejuiciada de textos que incluso se apartan de lo establecido por el canon. El objetivo no es crear un espacio de caos o libertinaje lector, sino constituir un espacio de reflexión y reorientación de intereses a partir de los comentarios de los compañeros y el docente que ya desde la perspectiva del estudiante se convierte en el modelo de lector.

En el caso específico que ocupa el estudio se aplicó dicha técnica con estudiantes del 5to de secundaria de dos instituciones particulares de la ciudad de Huaraz. Lo que se buscaba, específicamente, era determinar el grado de incidencia de dicha técnica en la motivación de los estudiantes en la revisión de textos recomendados por los compañeros del taller y el docente.

\section{MATERIALES Y MÉTODOS}

\section{Tipo de investigación}

El estudio realizado, por su finalidad es aplicado y por el diseño es de tipo cuasiexperimental con grupo control y experimental. Busca conocer la relación de incidencia entre la recomendación bibliográfica como técnica de animación por la lectura de los alumnos del 5to de secundaria de las instituciones educativas San Martín de Porras y Albert Einstein de Huaraz. La población está conformada por la totalidad de los estudiantes de 
las secciones ' $\mathrm{A}$ ' $\mathrm{y}$ ' $\mathrm{B}$ ' de las dos instituciones, que hacen un total de 120 estudiantes. La muestra está compuesta por 60 estudiantes de las sesiones ' $A$ ' de referidas instituciones.

La técnica que se utilizó en el pre test y post test para la recopilación de información fue la encuesta, y mediante el cuestionario se obtuvo información de la muestra, respecto a las dimensiones: intensidad de lectura, preferencias por tipos de texto y gusto por la lectura. El procesamiento de los datos se realizó a través del paquete estadístico SPSS versión 22.0 con un nivel de significancia con valor de $\mathrm{P}=0.05$.

\section{RESULTADOS}

En lo referente a la incidencia de la técnica de la animación a la lectura denominada la recomendación bibliográfica se ha podido evidenciar el incremento sustantivo en la motivación por la lectura a partir de la contrastación entre los resultados de los dos momentos diferentes: la etapa inicial y final:

\section{RESULTADOS DEL PRE TEST}

Figura 1: Intensidad de lectura. Describe la cantidad de libros leídos en los últimos seis meses, aparte de los textos obligatorios.

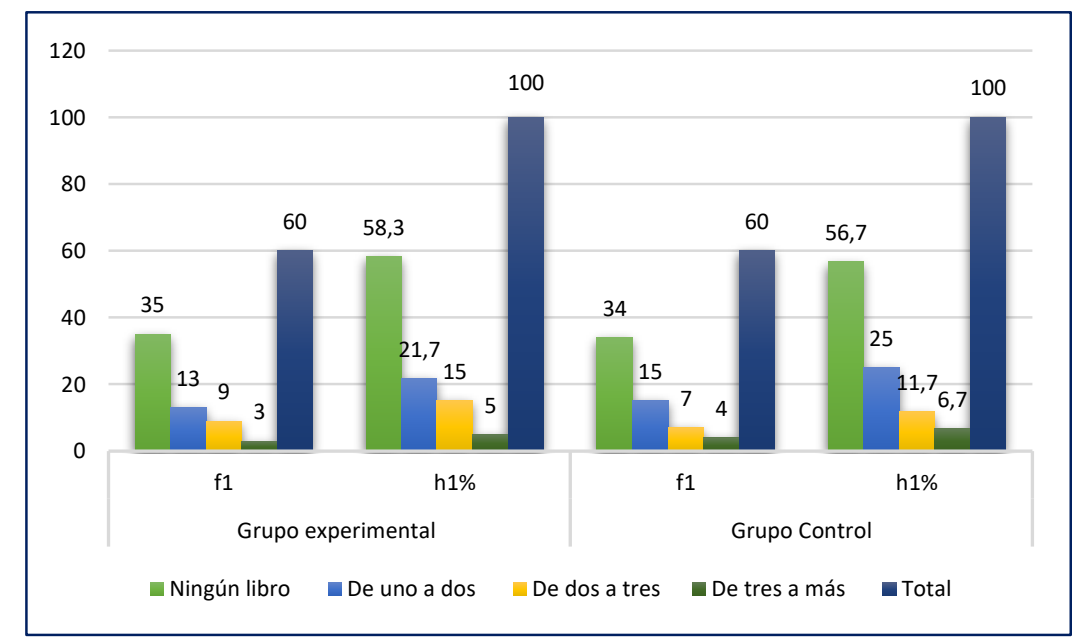

Como se puede observar el 58.3\% del grupo experimental manifiesta no haber leído ningún libro, a parte de las obligatorias en los últimos seis meses. El $21.7 \%$ declara haber leído de uno a dos; el 15\% de dos a tres; y el 5\% de tres a más. De la misma forma, el $56.7 \%$ del grupo control declara no haber leído ningún libro, el 25\% de uno a dos, el 11.7\% de dos a tres y el $6.7 \%$ de tres a más. De los porcentajes se evidencia la precariedad del interés por la lectura en ambos grupos de estudio. La cantidad de libros leídos implica un número reducido, demostrando de ese modo el escaso interés de los estudiantes por leer textos que no formen parte de la imposición académica requerida en su formación básica. 
Figura 2: Preferencias por tipos de texto. Describe las preferencias que tienen para leer según el tipo de texto.

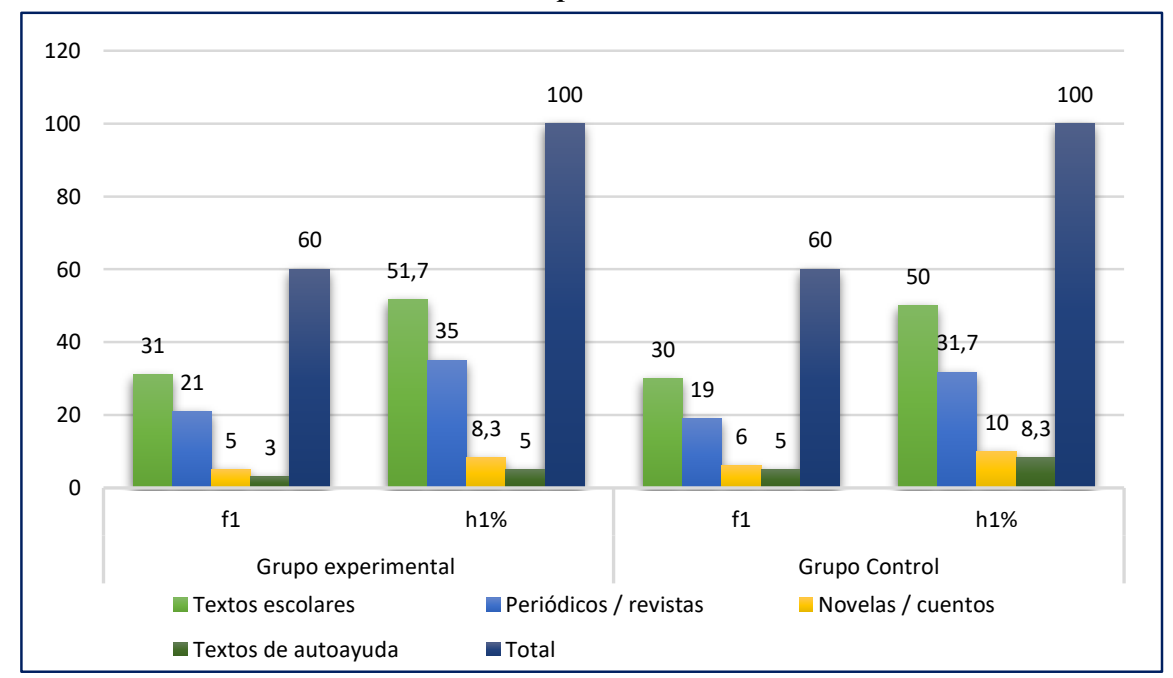

En la figura 2, a la pregunta, ¿qué tipo de libro le gusta leer más?, los encuestados del grupo experimental el 51.7\% respondió que le gusta leer más textos escolares, el 35\% periódicos y revistas, el $8.3 \%$ novelas y cuentos, y el 5\% textos de autoayuda. Mientras en el grupo control al 50\% de ellos le gusta leer textos escolares, al 31.7\% periódicos y revistas, al $10 \%$ novelas y cuento, y al $8.3 \%$ textos de autoayuda. La variedad de las preferencias en ambos grupos permite avizorar que, definitivamente, existe algún interés por diversos tipos de textos que no necesariamente formen parte de las lecturas obligatorias dentro en la formación básica académica de los alumnos. Sin embargo, también los datos permiten inferir que existe un porcentaje considerable de aquellos que solo se circunscriben a los textos escolares como sus preferidos a la hora de elegir un tipo de texto; ello indica la falta de motivación de los alumnos hacia textos de distinta naturaleza.

Figura 3: Gusto por la lectura. Describe sobre cuánto les gusta leer

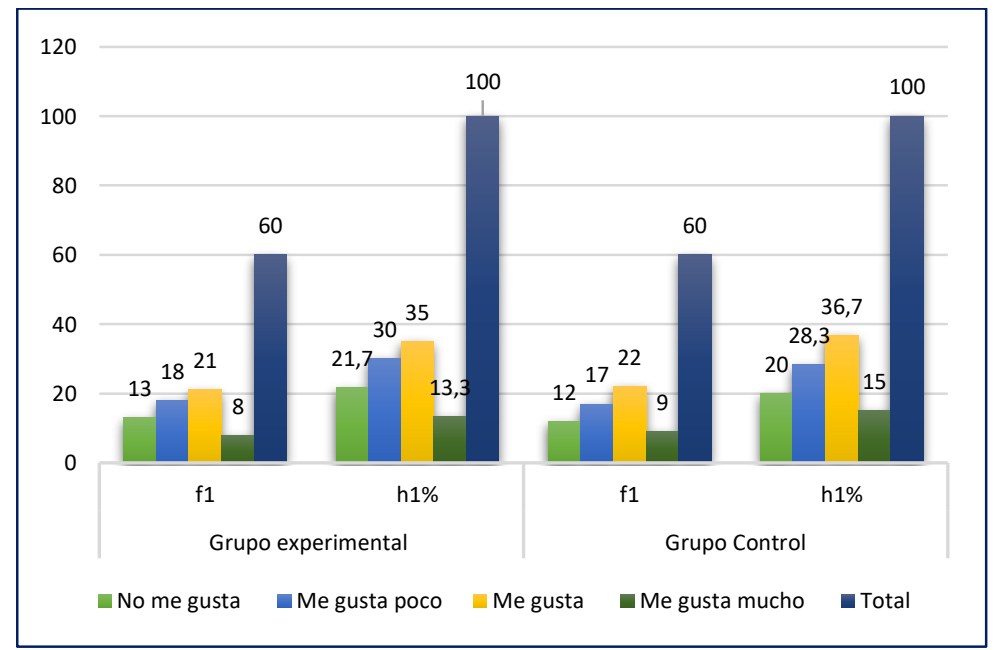


En la figura 3 los resultados de la encuesta por el gusto hacia la lectura que expresan los alumnos identificados como grupo experimental se indica que al $21.7 \%$ no le agrada la lectura, al 30\% le agrada poco, al 30\% le agrada y al $13.3 \%$ lo prefiere. En tanto, al $20 \%$ del grupo control no le gusta, al $28.3 \%$ le gusta poco, al 36.7 le gusta y al $15 \%$ le gusta mucho. Estos datos son expectantes con relación a la investigación realizada, pues evidencian el valor que virtualmente tiene la lectura para los estudiantes; probablemente los que afirman que no les agrada la lectura evidencian problemas de comprensión o problemas en la metodología de comprensión lectora que utilizan para desentrañar el contenido de un texto. La dispersión de los porcentajes resulta interesante, en la medida que les permite determinar grados de progresión entre los alumnos a partir de un trabajo dedicado a elevar la capacidad lectora de los estudiantes.

Figura 4: Describe las razones del por qué leer un texto

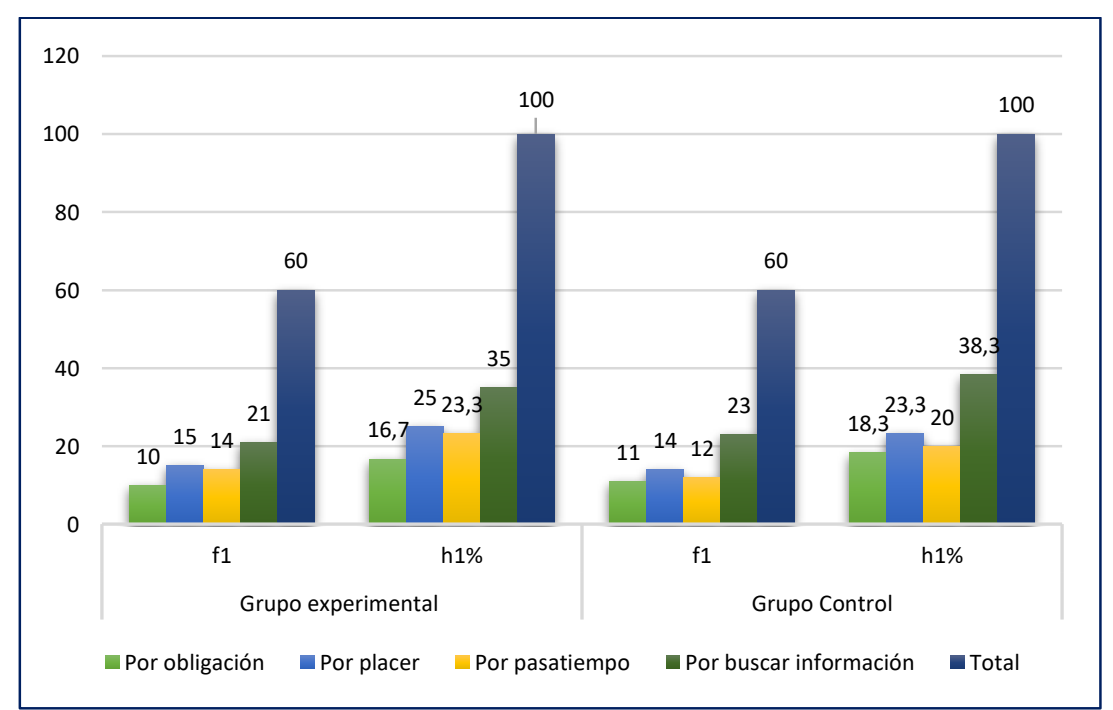

De los resultados de la figura 4, se valora que el 16.7\% del grupo experimental considera que lee por obligación, el $25 \%$ por placer, el $23.3 \%$ por pasatiempo y el $35 \%$ por buscar información. En tanto, los resultados del grupo control son similares, en que el $18.3 \%$ manifiesta leer por obligación, el $23.3 \%$ por placer, el $20 \%$ por pasatiempo y el $38.3 \%$ por buscar información. Tal como se puede apreciar en los datos porcentuales, la lectura es para muchos estudiantes, en ambos grupos, solo una técnica para recabar información, en ese sentido cumpliría solo una función utilitaria. No obstante, también resultan apreciables los porcentajes referidos a los que la realizan por disfrute. Se considera preocupante los porcentajes que indican una lectura motivada solo por la imposición y no por el placer que se deriva de ella. 


\section{RESULTADOS DEL POST-TEST}

Figura 5: Intensidad de lectura. Describe la cantidad de libros leídos en los últimos seis meses, aparte de los textos obligatorios.

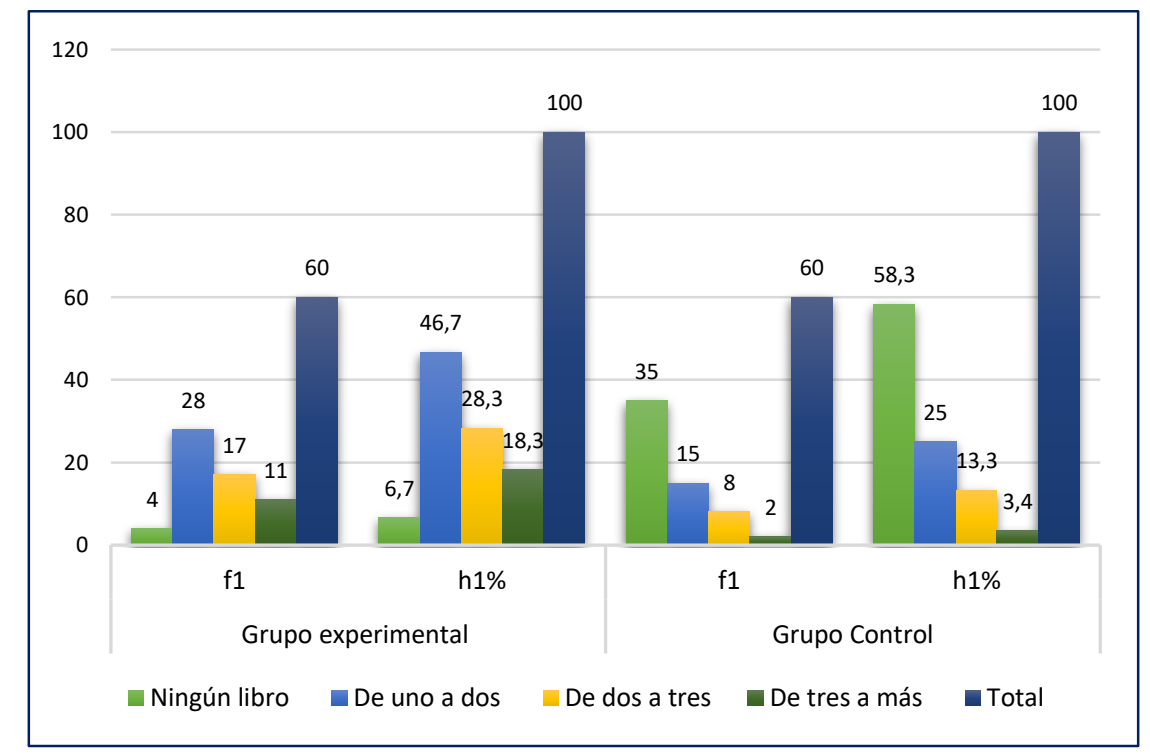

Figura 5. Número de libros leídos en los último seis meses.

De los resultados de la figura 5, se obtuvo que el 6.7\% del grupo experimental asegura no haber leído algún libro a parte de las obligatorias en estos últimos seis meses; mientras en el grupo control asciende al 58.3\%. Del mismo modo, en el grupo experimental el $46.7 \%$ de los encuestados indica haber leído de uno a dos libros en el semestre y en el grupo control, solo el 25\%. Asimismo, el 28.3\% señaló haber leído de dos a tres libros en el grupo experimental y en el grupo control el 13.3\%. Finalmente, el $18.3 \%$ del grupo experimental asegura haber leído de tres a más libros; mientras en el grupo control solo 3.4\%. Los datos revelan, claramente, la mejora significativa en los estudiantes del grupo experimental, pues las evidencias señalan que ellos han leído más libros que los estudiantes del grupo control. Se afirma, entonces, que la estrategia propuesta conllevó a elevar la proclividad en favor de la lectura. 
Figura 6: Preferencias por tipos de texto. Describe las preferencias para leer según el tipo de

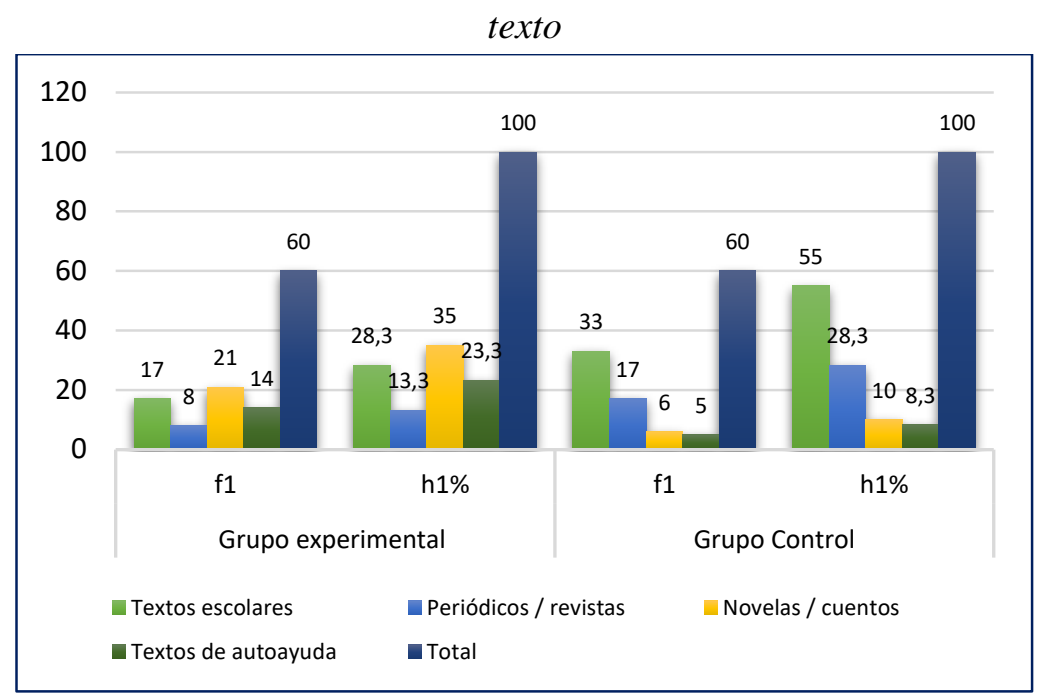

Según los resultados en la figura 6 se observa que el grupo experimental presenta diferencias significativas respecto a lo obtenido por el grupo control. En el grupo experimental hay una tendencia positiva a la diversificación en la lectura de textos, siendo así que $28.3 \%$ indica gustarle leer textos escolares; el 13.3\%, periódicos y revistas; el 35\%, novelas y cuentos; y el $23.3 \%$ textos de autoayuda. En cambio, en el grupo control la tendencia es a la «homogenización», porque más de la mitad de ellos $(55 \%)$ indica tener gusto por texto escolares, el $28.3 \%$ periódicos y revistas, el $10 \%$ novelas y cuentos, el $8.3 \%$ textos de autoayuda. La diversificación en el gusto de lectura en el grupo experimental es mayor con respecto al grupo control; ello revela que los estudiantes del grupo experimental han encontrado, con las técnicas de animación aplicada, motivación para interesarse en textos de diferente naturaleza.

Figura 7: Gusto por la lectura. Describe sobre cuánto les gustar leer

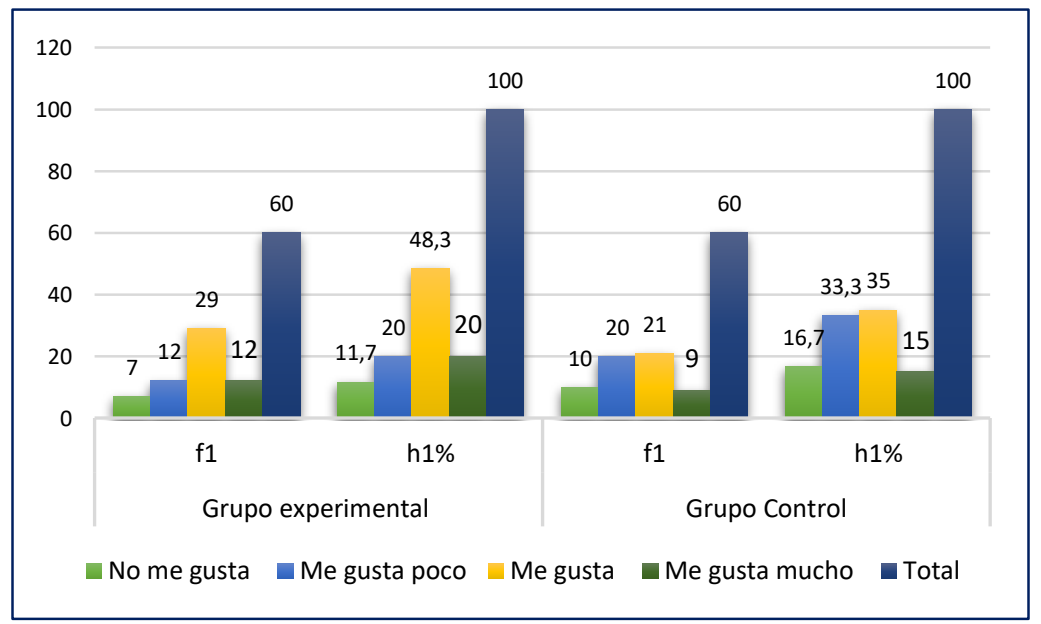


Según los resultados de la figura 7 , respecto a los niveles del gusto por la lectura, se indica que el $11.7 \%$ del grupo experimental afirma que no le gusta leer; mientras al 20\% le gusta poco. Asimismo, el $48.3 \%$ indica que le gusta y el $20 \%$ le agrada mucho. En contraste, en el grupo control $16.7 \%$ indica que no le gusta leer, el 33.3\% le gusta poco, el 35\% le gusta y al $15 \%$ le gusta mucho. El porcentaje hallado en el grupo experimental del $48.3 \%$ y $20 \%$ en favor del gusto por la lectura revela un mejoramiento significativo del interés por la actividad al haber aplicado esta técnica en contraste con lo que se revela en el grupo control donde el $50 \%$ (35\% y $15 \%$ ) tiene desarrollado el gusto por leer.

Figura 8: Describe las razones del por qué leer un texto

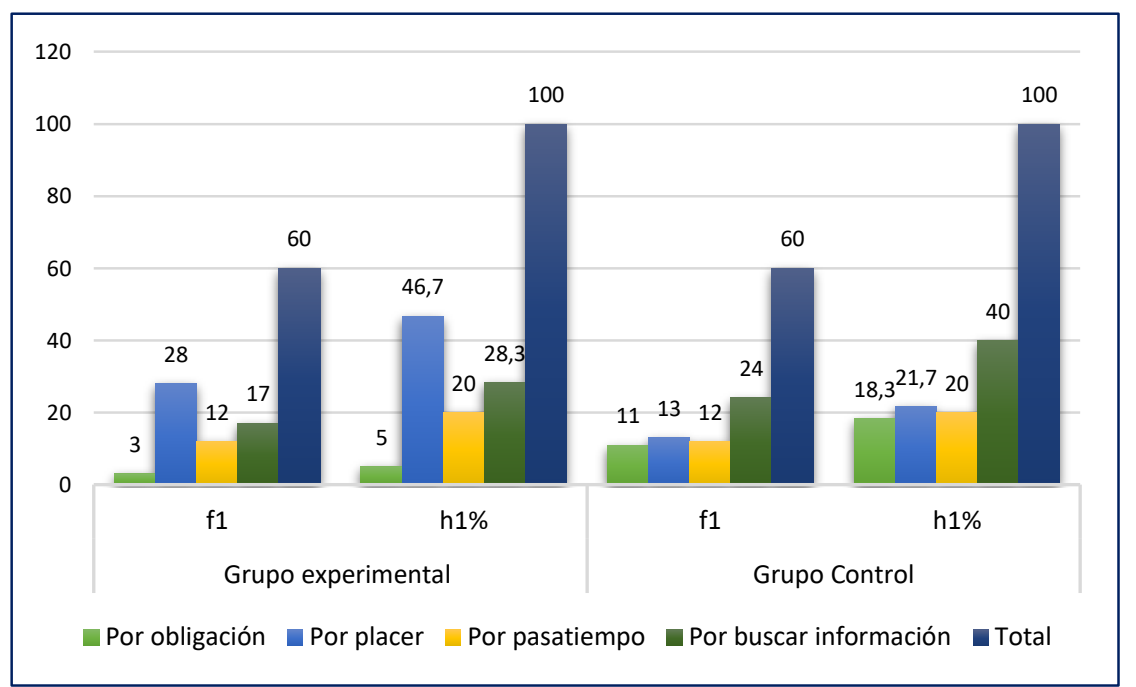

De los resultados de la figura 8, se afirma que el 5\% del grupo experimental asegura leer por obligación, mientras en el grupo control esta cifra aumenta considerablemente llegando al $18.3 \%$. Del mismo modo se demuestra que en el grupo experimental $46.7 \%$ indica leer por placer y en el grupo control el 21.7\%. El 20\% señala leer por pasatiempo en el grupo experimental y en el grupo control el 20\%. Finalmente, el $28.3 \%$ asegura leer por buscar información, en el primer grupo y en el segundo, 40\%. El incremento del grupo experimental con relación a la lectura realizada por placer en contraste con el $21.7 \%$ del grupo control, muestra claramente la conveniencia de la técnica de recomendación bibliográfica para generar mayor expectativa en los estudiantes, demostrando con ello el valor de la esta propuesta. 


\section{DISCUSIÓN DE RESULTADOS}

La investigación sobre la incidencia de la recomendación bibliográfica como técnica de animación a la lectura de los alumnos del 5to de secundaria de las instituciones educativas privadas San Martín de Porras y Albert Einstein de la ciudad de Huaraz reporta que existe influencia significativa de la variable independiente a la variable dependiente. En general, el coeficiente de correlación es positivo debido a que alcanza más del 50\% el signo bilateral .000 de un total de 60 estudiantes intervenidos. Ello evidencia la aplicabilidad de dicha técnica en los ámbitos académicos escolares.

A continuación, se desarrolla en forma concreta las diferentes dimensiones que alcanzan cambios sustanciales en la intensidad lectora, el cambio sustancial por las preferencias según tipo de texto, el gusto por la lectura y los motivos del por qué leer. Es evidente que la aplicación de la técnica de animación a la lectura ha de recaer forzosamente en una persona concreta, preparada, dispuesta a realizar su trabajo con entusiasmo, objetivos claros y confianza (Sarto, 1993; Bermúdez y Núñez, 2012). Esta persona ha de ser el docente - animador, y sobre él recae la disposición programática, es decir, el establecimiento de los distintos objetivos, la determinación del número de animaciones necesarias para alcanzar los objetivos fijados, la preparación previa de las estrategias, la selección de los libros adecuados para cada caso, la creación del ambiente favorable (Bermúdez y Núñez, 2015). Así pues, el docente guía y animador debe estar dotado de cualidades altamente comunicativas.

La comparación de los resultados de las figuras 1 y 5 de la cantidad de libros leídos en los últimos seis meses entre la etapa del pre test y el post test demuestra la incidencia positiva de la recomendación bibliográfica como técnica de animación a lectura. Los resultados en el pre test evidencian que la cantidad de libros leídos es ínfimo, demostrando el escaso interés por la lectura; mientras en el post test hay un aumento sustancial, lo que permite confirmar que la estrategia seguida conlleva a mayor predilección por esta actividad. Demuestra que la recomendación bibliográfica en las actividades académicas en el aula contribuye al fortalecimiento del hábito lector, esa destreza lingüística que requiere de cierta frecuentación de la lectura para afianzarse (Cerrillo, 2004:256). Si bien es cierto, el incremento no resulta sobreabundante, se puede percibir, sin embargo, el rasgo de influencia sintomático valioso que ofrece la técnica aplicada. 
En las figuras 2 y 6 refleja el cambio sustancial en el post test por las preferencias de lectura según tipo de texto respecto al pre test (textos escolares, revistas/periódicos, novelas/cuentos y textos de autoayuda). En el pre test se evidencia que existe un interés mayoritario por textos escolares, que forman parte de lecturas obligatorias en la formación académica de los estudiantes. Estos datos demuestran la falta de motivación por otros tipos de textos. En el post test se experimenta la diversificación en el gusto según el tipo de texto, ello revela que el grupo experimental de alumnos ha encontrado motivación y gusto por explorar otras posibilidades de contenido la lectura según los tipos de texto, lo anterior indica la incidencia de la recomendación bibliográfica como técnica de animación a lectura. Esta actitud positiva hacia diversos tipos de textos; significa, no solo el aprovechamiento del tiempo libre, sino también la adquisición de una valiosa experiencia que favorece su desarrollo como lectores competentes (Mullis y otros, 2006). Asimismo, tener en cuenta que el contenido, la organización y el estilo que pudieran ser característicos de un género de texto en particular influyen en la manera en la que el lector aborda la lectura (Graesser, Golding y Long, 2000, citado en Mullis y otros, 2006).

Respecto el gusto por la lectura, los resultados de la figura 3 del pre test y la 7 del post test revelan con claridad una diferencia entre el pre prueba y la post prueba. En la primera etapa hay una tendencia importante hacia la actitud negativa por la lectura. En el post test hay un cambio sustancial del interés por la lectura. Esto demuestra que la técnica aplicada hace que el estudiante adquiera un distinto «comportamiento lector», pues desarrolla actitudes positivas hacia la lectura y leen para el disfrute personal (García, 2004). El cambio significativo hacia el gusto por la lectura, si se sostiene a lo largo del tiempo, crea en los estudiantes lectores activos y eficientes, capaces de enfrentarse a textos de creciente dificultad según ciertos niveles de progresión convirtiéndolos en lectores autónomos. (López, 2004 y García, 2004). Y, «han de percibirse a sí mismos como personas competentes, que con las ayudas y recursos necesarios, podrán tener éxito y apropiarse de ese instrumento que les será tan útil para la escuela y para la vida» (Solé, 1995: 4).

Finalmente, en las figuras 4 y 8 la comparación de resultados entre el pre test y el post test de las razones del por qué leen un texto, se precisa que en la pre prueba un porcentaje significativo considera que lo hace por obligación y no por placer o por buscar información. En tanto, en la post prueba se nota el incremento en el grupo experimental con relación a la lectura que se realiza por placer y por la búsqueda de información. Los resultados permiten interpretar que el uso de la técnica de recomendación bibliográfica 
contribuye a la realización personal del potencial de individuo en el seno de una sociedad instruida (Mullis y otros, 2006). También permite generar en los alumnos un deseo sano por la lectura, pues esta no debe ser considerada una obligación. Por tanto, la lectura tiene una dimensión personal, lúdica y placentera que, una vez conocida, acompaña a las personas a lo largo de toda su vida, sin dejar nunca de alimentarse y de gratificar a quien la ejerce (Solé, 1995: 4).

\section{CONCLUSIONES}

- La recomendación bibliográfica como técnica de animación a la lectura contribuye al fortalecimiento del hábito lector en los estudiantes del 5to de secundaria de la I.E.P. Albert Einstein de Huaraz y la I.E.P. San Martín de Porras tal como lo revelan los resultados del post-test.

- La recomendación bibliográfica como técnica de animación a la lectura contribuye a elevar los niveles de diversificación del gusto lector en los estudiantes del 5to de secundaria de la I.E.P. Albert Einstein de Huaraz y la I.E.P. San Martín de Porras tal y como lo revelan los resultados del post-test.

- La recomendación bibliográfica como técnica de animación a la lectura contribuye a que el estudiante desarrolle actitudes positivas hacia la lectura y el disfrute personal de los estudiantes del 5to de secundaria de la I.E.P. Albert Einstein de Huaraz y la I.E.P. San Martín de Porras.

- La recomendación bibliográfica como técnica de animación a la lectura promueve a que se asuma la lectura como una actividad placentera que permita alcanzar la realización personal cognitiva en los estudiantes del 5to de secundaria de la I.E.P. Albert Einstein de Huaraz y la I.E.P. San Martín de Porras.

\section{REFERENCIAS BIBLIOGRAFÍCAS}

Anderson, R. y Pearson, D. (1984). A schema-theoretic view of basic processes in reading comprehension. En P.D. Pearson (ed.). Handbook of reading research (pp. 255-291). White Plains, Nueva York: Longman.

Bermúdez, M. y Núñez, M. (2012). Canon y educación literaria. Barcelona: Octaedro.

Bobbio-Älvarez, R. (2014). Diagnóstico de la eficacia del plan lector en alumnos de educación secundaria. Piura.

Cerrillo, P. (2004). Promoción y animación a la lectura. En: López A. y Encaba, E. (coord.) Didáctica de la literatura, el cuento, la dramatización y la animación a la lectura. Barcelona: Octaedro. 
Domech, C. et. al. (1994). Animación a la lectura: ¿Cuántos cuento cunetas tú? Madrid: Popular

García, G. (2004). La literatura infantil y juvenil como marco de referencia a la animación a la lectura. En: López A. y Encaba, E. (coord.) Didáctica de la literatura, el cuento, la dramatización y la animación a la lectura. Barcelona: Octaedro.

Higuera, G. (2016). De la estética de la recepción a la animación a la lectura literaria en espacios no convencionales. En: La Palabra. $\mathrm{N}^{\circ} 28$. Tunja

López, M. y otros. (2004). El taller como estrategia de animación a la lectura. En: López, A y Encabo, E. (coord.) Didáctica de la literatura, el cuento, la dramatización y la animación a la lectura. Barcelona: Octaedro.

Millán, J. (2000). La lectura y la sociedad del conocimiento. Pamplona: Gobierno de Navarra. Departamento de Educación.

Mullis, I. y otros. (2003). PIRLS 2001 international report: IEA's study of reading literacy achievement in primary school in 35 countries. Chestnut Hill, Massachussets: Boston.

Mullis, I. y otros. (2006). Estudio internacional de progreso en comprensión lectora. Traducido por Mercedes Núñez. Boston: Ministerio de Educación y Ciencia - Secretaría General de Educación - Instituto Nacional de Evaluación y Calidad del Sistema Educativo (INECSE).

Poslaniec, C. (1990). Animación a la lectura. promolej Francia Clij

Sarto, M. 1993. La animación a la lectura. Madrid: SM.

Sedano, M. (2015). Leer en el aula. En: Revista de Ciencias humanas y sociales. $\mathrm{N}^{\circ} 6$. España.

Solé, I. 1995. "Lectura y vida. El placer de leer”. En: Revista latinoamericano de lectura. Año 16, $\mathrm{N}^{\circ}$ 3. Barcelona.

Devetach, L. (2008). La construcción del camino lector. Córdoba: Comunicarte.

Cassany, D. y otros. (2003). Enseñar lengua. Barcelona: GRAÓ.

Yepes, L. (2001) Acciones de promoción de lectura en COMFENALCO-Antioquia. En Juan Pablo Hernández Carvajal, Animación y promoción de la lectura: consideraciones y propuestas. Colombia: COMFENALCO, Colección fomento de la lectura; $\mathrm{N}^{\circ} 3$. 